\title{
Anatomical differences in patients with lumbosacral transitional vertebrae and implications for minimally invasive spine surgery
}

\author{
Darnell T. Josiah, MS, MD, ${ }^{1}$ SoHyun Boo, MD, ${ }^{2}$ Abdul Tarabishy, MD, ${ }^{2}$ and Sanjay Bhatia, MBBS ${ }^{1}$ \\ Departments of ${ }^{1}$ Neurosurgery and ${ }^{2}$ Radiology, West Virginia University School of Medicine, Morgantown, West Virginia
}

OBJECTIVE The objective of this study was to investigate the neurovascular and anatomical differences in patients with lumbosacral transitional vertebrae (LSTV) and the associated risk of neurovascular injury in minimally invasive spine surgery.

METHODS The authors performed a retrospective study of CT and MR images of the lumbar spine obtained at their institution between 2010 and 2014. The following characteristics were evaluated: level of the iliac crest in relation to the L4-5 disc space, union level of the iliac veins and arteries in relation to the L4-5 disc space, distribution of the iliac veins and inferior vena cava according to the different Moro zones (A, I, II, III, IV, P) at the L4-5 disc space, and the location of the psoas muscle at the L4-5 disc space. The findings were compared with findings on images obtained in 28 age- and sex-matched patients without LSTV who underwent imaging studies during the same time period.

RESULTS Twenty-eight patients (12 male, 16 female) with LSTV and the required imaging studies were identified; 28 age- and sex-matched patients who had undergone CT and MRI studies of the thoracic and lumbar spine imaging but did not have LSTV were selected for comparison (control group). The mean ages of the patients in the LSTV group and the control group were 52 and 49 years, respectively. The iliac crest was located at a mean distance of $12 \mathrm{~mm}$ above the L4-5 disc space in the LSTV group and $4 \mathrm{~mm}$ below the L4-5 disc space in the controls. The iliac vein union was located at a mean distance of $8 \mathrm{~mm}$ above the L4 -5 disc space in the LSTV group and $2.7 \mathrm{~mm}$ below the L4-5 disc space in the controls. The iliac artery bifurcation was located at a mean distance of $23 \mathrm{~mm}$ above the L4-5 disc space in the LSTV group and $11 \mathrm{~mm}$ below the L4-5 disc space in controls. In patients with LSTV, the distribution of iliac vein locations was as follows: Zone A, 7.1\%; Zone I only, 78.6\%; Zone I encroaching into Zone II, 7.1\%; and Zone II only, 7.1\%. In the control group, the distribution was as follows: Zone A only, 17.9\%; Zone A encroaching into Zone I, 75\%; and Zone I only, 7.1\%. There were no iliac vessels in Zone II in the control group. The psoas muscle was found to be rising away laterally and anteriorly from the vertebral body more often in patients with LSTV, resulting in the iliac veins being found in the "safe zone" only $14 \%$ of the time, greatly increasing the risk of vascular injury.

CONCLUSIONS In patients with LSTV, the iliac crest is more likely to be above the L4-5 disc space, which increases the technical challenges of a lateral approach. The location of the psoas muscle rising away laterally and ventrally in patients with LSTV compared with controls and with the union of the iliac veins occurring more often above the L4-5 disc space increases the risk for iatrogenic vascular injury at the L4-5 level in this patient population.

https://thejns.org/doi/abs/10.3171/2016.6.SPINE1691

KEY WORDS transitional vertebrae; minimally invasive spine surgery; extreme lateral interbody fusion; complication; lumbar

$\mathrm{L}$ OW-BACK pain affects millions of people worldwide, with an estimated $80 \%$ of the adult population experiencing low-back pain at some time..$^{1,10}$ Lumbosacral transitional vertebra refers to an elongation of the transverse process of the L-5 vertebra with a total or partial, unilateral or bilateral fusion to the sacrum. ${ }^{7,13}$ This condition is the most common congenital anomaly of the lumbosacral spine, with a prevalence that varies between $4 \%$ and $35 \%$ in various reports. ${ }^{2,17}$

The presence of a transitional vertebra was postulated as a cause of low-back pain in 1917 by Bertolotti. ${ }^{17} \mathrm{~A}$ population-based study that included 5500 individuals and examined the prevalence and relationship of lumbosacral transitional vertebrae (LSTV) to low-back pain in the Chi-

ABBREVIATIONS IVC = inferior vena cava; LSTV = lumbosacral transitional vertebrae.

SUBMITTED January 18, 2016. ACCEPTED June 20, 2016.

INCLUDE WHEN CITING Published online September 23, 2016; DOI: 10.3171/2016.6.SPINE1691. 
nese population found a prevalence of $15.8 \%$, with a significant association of low-back pain and gluteal pain with the degree of osseous fusion. ${ }^{18}$ Luoma et al. ${ }^{13}$ found an increased risk of degenerative changes in the disc above the transitional vertebra among young men, and other studies $^{15,22}$ have shown an acceleration of disc and facet joint degeneration at the superjacent level, L4-5.

Accurate identification of LSTV is important because incorrect identification can lead to wrong-level surgeries or procedural errors. Historically, LSTV have been identified using lateral and Ferguson films. ${ }^{12}$ Castellvi et al. ${ }^{6}$ characterized 4 types of LSTV using radiographs based on morphological characteristics. Type I includes Ia (unilateral) or Ib (bilateral) dysplastic transverse process(es) measuring at least $19 \mathrm{~mm}$ in craniacaudal dimensions. Type II includes incomplete IIa (unilateral) or IIb (bilateral) lumbarization/sacralization of the transverse process with a diarthrodial joint with the sacrum. Type III includes IIIa (unilateral) or IIIb (bilateral) lumbarization/ sacralization with complete osseous fusion. Type IV is a unilateral Type II with a contralateral Type III lumbarization/sacralization.

If plain radiographs are not available, as is often the case when lumbar MRI studies are ordered, the cervicothoracic scout images are used for numbering the vertebral levels. Paraspinal structures, such as the aortic bifurcation, right renal artery origin, conus medullaris, and iliolumbar ligament, have been reported to aid in the identification of vertebral levels., ${ }^{919}$ The iliolumbar ligament, which was once considered the most reliable anatomical landmark, was found in recent studies to have multiple origins, sometimes originating from L-4 or S-1. ${ }^{9}$

Lumbar fusion with or without decompression is often used for the treatment of symptomatic lumbar disc degenerative disease when conservative therapy has failed. Open surgical approaches to lumbar fusion such as ALIF (anterior lumbar interbody fusion) or PLIF (posterior lumbar interbody fusion) often require significant muscle and ligament dissection, mobilization of vascular structures, and retraction of neural elements. Minimally invasive approaches, such as the lateral transpsoas approach, were developed in an attempt to minimize the morbidity and complications associated with the traditional approaches, but they require good intraoperative fluoroscopy and careful attention to preoperative imaging studies to evaluate for anomalous anatomy. ${ }^{4,16}$

We assessed the anatomy that is particularly relevant for minimally invasive approaches to the lumbosacral spine in the presence of a lumbosacral transitional vertebra. This anomaly affects the L5-S1 level with direct consequences for the L4-5 level and minimally invasive ALIF or lateral transpsoas approach, which are commonly used for stabilization, fusion, and indirect decompression of the L4 -5 foramina.

\section{Methods}

We conducted a retrospective review of $\mathrm{CT}$ and MR images of the lumbar spine obtained between 2010 and 2014 in patients with lumbarization or sacralization of the vertebra and compared the findings with those obtained in age- and sex-matched controls. The study was approved by the institutional review board of West Virginia University School of Medicine.

\section{Study Population}

All initial CT scans obtained in patients with a lumbosacral transitional vertebra during the study period were reviewed. A total of 28 patients had a lumbosacral transitional vertebra evident on these imaging studies and also had a corresponding MRI study of the lumbosacral spine and were included in the study. This group consisted of 12 male and 16 female patients. Twenty-eight age- and sex-matched patients with nonspecific back and radicular pain who had also undergone CT and MRI studies of the thoracic and lumbar spines were selected as controls. The thoracic vertebra and the number of ribs were counted using CT scans that included both the thoracic and lumbar spine. The number of non-rib-bearing vertebrae was then noted for patients from both the LSTV group and the control cohort.

\section{Imaging Analysis}

Imaging studies were reviewed by the authors for the following characteristics: 1) level of the iliac crest in relation to the L4-5 disc space, 2) union of the iliac veins and arteries, 3) size of the L4-5 disc space, 4) relationship of the iliac veins and the inferior vena cava (IVC) to the L4-5 disc space in terms of Moro zones, and 5) location of the psoas muscle with respect to the Moro zones. Coronal MR images were used to define the height of the iliac crest with respect to the middle of the L4-5 disc space. Sagittal MR images were used to measure the disc height, and axial MR images were used to measure the sagittal and transverse disc diameters, the relationship of the vascular and neural structures to the L4-5 disc space, and the safe working zones. The Moro classification divides the area between the anterior edge of the vertebral body and the posterior edge of the vertebral body into 4 zones. The most anterior zone is defined as Zone I, the second most anterior zone as Zone II, the third as Zone III, and the most posterior area as Zone IV. Zone P refers to the area posterior to the posterior edge of the vertebral body, and Zone A refers to the area anterior to the anterior edge of the vertebral body..$^{14}$

\section{Statistical Analysis}

Two-tailed t-tests were performed using GraphPad Prism version 6 for Mac. The means were compared between the groups and the mean values with the standard error of the mean are presented.

\section{Results}

Images obtained in 28 patients with LSTV and 28 age-matched patients without LSTV were analyzed. Each group included 12 male and 16 female patients. The patients' mean age at the time of imaging was $51.79 \pm 2.96$ years in the LSTV group and $48.93 \pm 2.8$ years in the control group (no significant difference, $\mathrm{p}=0.4862$ ).

The level of the iliac crest was measured on coronal MR images in relation to the center of the L4-5 disc space. Negative values of the measurements indicate that 
the iliac crest was found above the center of the L4-5 disc space, while positive numbers indicate that the horizontal level of the iliac crest was below the center of the L4-5 disc space. The mean distance to the iliac crest in patients with LSTV was $-12.05 \pm 1.738 \mathrm{~mm}$, whereas for controls it was $4.055 \pm 2.380 \mathrm{~mm}(\mathrm{p}<0.0001$, Fig. 1$)$.

The location of the union of the iliac veins was analyzed on axial and sagittal MR images in relation to the center of the L4-5 disc space. Negative numbers in millimeters indicate that the iliac veins merged above the center of the L4-5 disc space, while positive numbers indicate that the veins merged below the center of the L4 -5 disc space. The mean distance to the iliac vein union in patients with LSTV was $-8.189 \pm 1.732 \mathrm{~mm}$, whereas for the control group it was $2.672 \pm 2.417 \mathrm{~mm}$ ( $\mathrm{p}=0.0006$, Fig. 2).

The location of the bifurcation of the iliac arteries was analyzed on axial and sagittal MR images in relation to the center of the L4-5 disc space. Negative numbers in millimeters indicate that the iliac arteries bifurcated above the center of the L4-5 disc space, while positive numbers indicate that the arteries split below the center of the L4-5 disc space. The mean location of the iliac artery bifurcation in patients with LSTV was $-23.15 \pm 2.068$, whereas for the control group it was $11.29 \pm 3.418$ ( $p<$ 0.0001, Fig. 3).

Using axial MR images, the L4-5 disc space was divided into the 6 Moro zones and the location of the iliac veins and/or IVC was analyzed to determine what zone(s) they encroached or occupied. In patients with LSTV, the distribution by zone was as follows: Zone A, 7.1\%; Zone I only, 78.6\%; Zone I encroaching into Zone II, 7.1\%; Zone II only $7.1 \%$. The veins were never found in Zones III, IV, or P in the LSTV group (Fig. 4 left). In the control group, the distribution was: Zone A only, 17.9\%; Zone A encroaching into Zone I, 75\%; Zone I only, 7.1\%. The veins were never found to enter or encroach into Zones II, III, IV, or P in the patients without LSTV (Fig. 4 right).

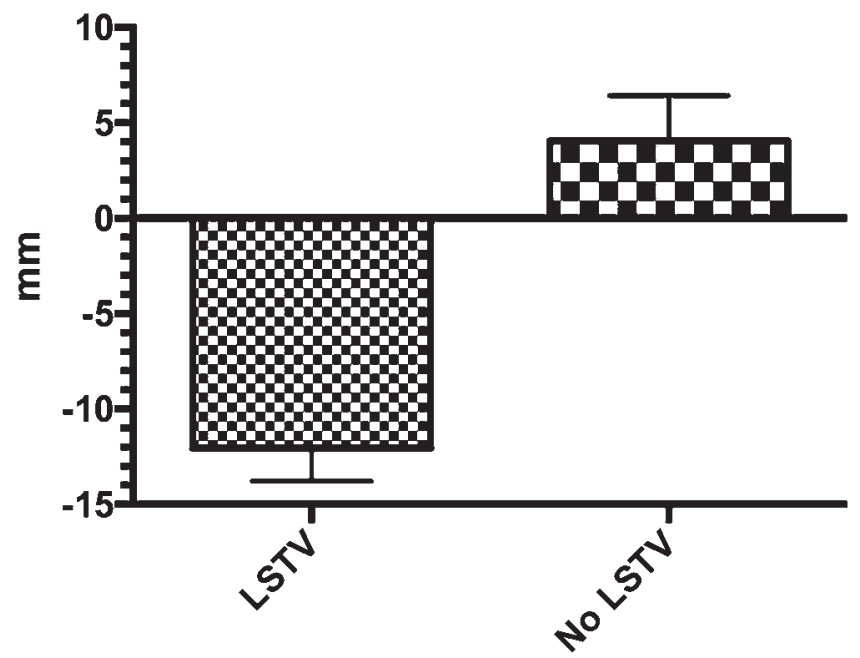

FIG. 1. Horizontal level of the iliac crest in relation to the L4-5 disc space in patients with LSTV and age- and sex-matched controls (No LSTV). Mean values are presented with SEM (error bars). Negative values on the $y$-axis indicate distance in millimeters above the center of the L4-5 disc space; positive values indicate distance below it.

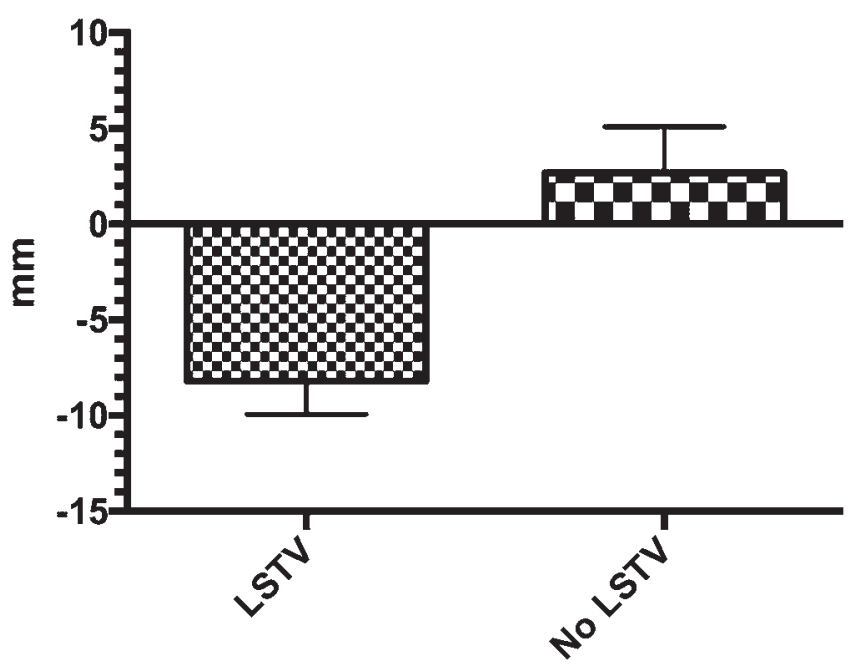

FIG. 2. Location of union of iliac veins in relation to the L4-5 disc space in patients with LSTV and age- and sex-matched controls. Mean values are presented with SEM (error bars). Negative values on the $y$-axis indicate distance in millimeters above the center of the L4-5 disc space; positive values indicate distance below it.

The locations of the left and right psoas muscles were analyzed on axial MR images to determine which zone(s) they occupied and apposition to the disc. In patients with LSTV, the relationship of the left psoas muscle to the Moro zones was as follows: none (no apposition) in 3.6\%; none and Zone A (no apposition and anterior to the disc) in $50 \%$; Zones A, II, and III in 24.1\%; Zones A, I, II, and III in 3.6\%; Zones I, II, and III in 7.1\%; and Zones II and III in $7.1 \%$ (Fig. 5A). The relationship with respect to the right psoas muscle in patients with LSTV was as follows: none (no apposition) in 3.6\% of patients; none and Zone A (no apposition and anterior to the disc) in 35.7\%; Zones A and II in 7.1\%; Zones A, II, and III in 35.7\%; Zones I, II, and III in 10.7\%; Zones II and III in 3.6\%; and Zone III only in $3.6 \%$ (Fig. 5B).

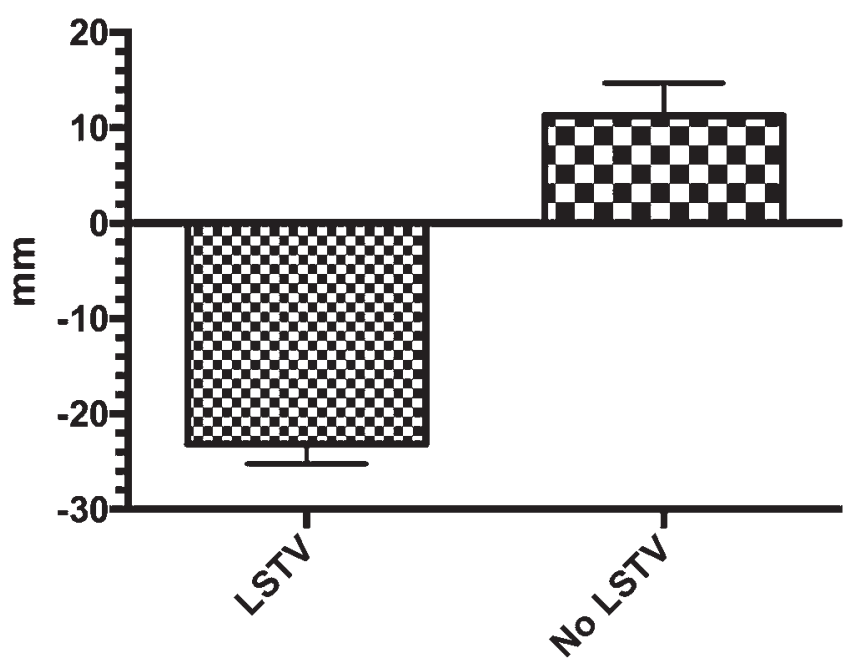

FIG. 3. Bifurcation of the iliac arteries in relation to the center of the L4-5 disc space. Mean values are presented with SEM (error bars). Negative values on the $y$-axis indicate distance in millimeters above the center of the L4-5 disc space; positive values indicate distance below it. 

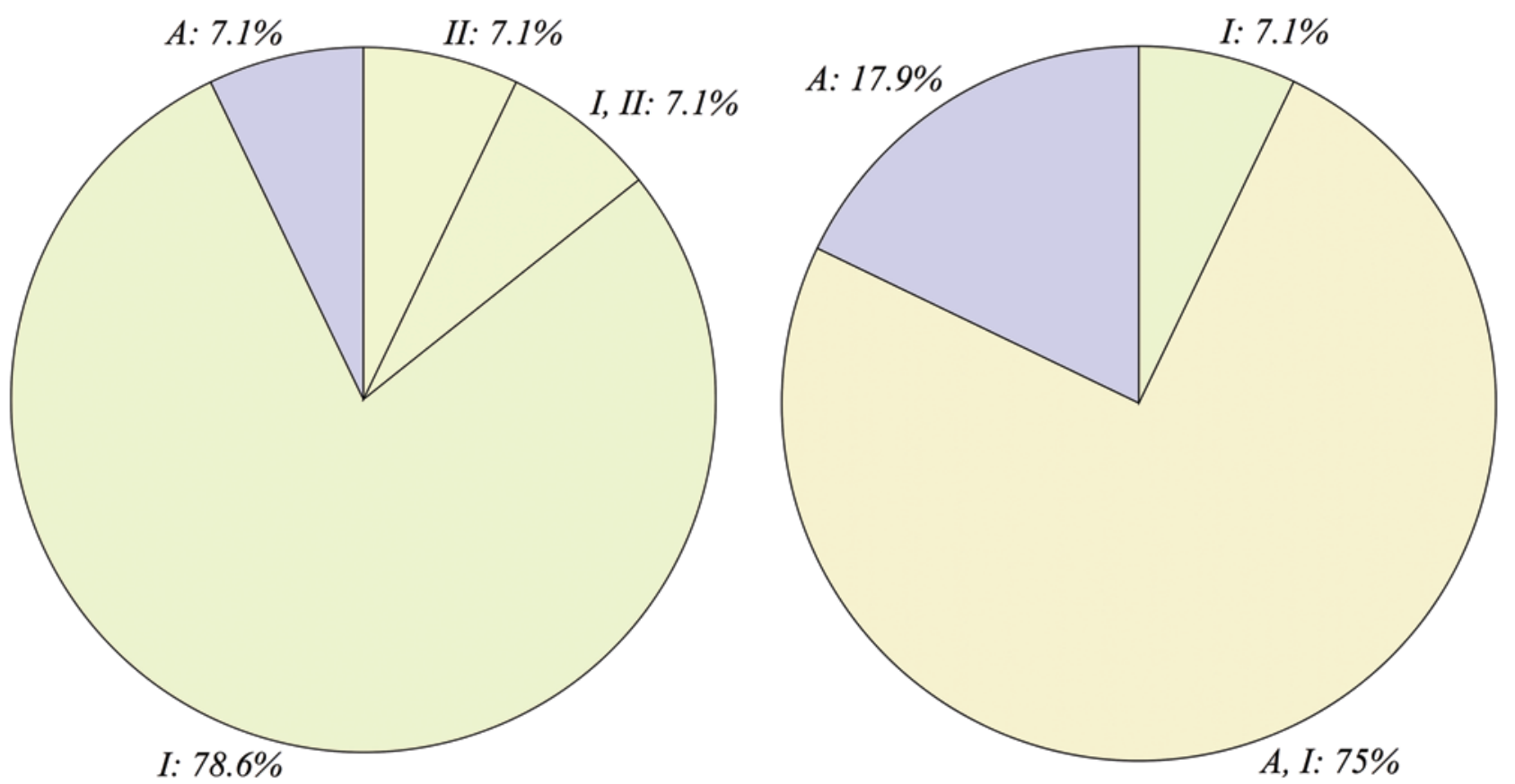

FIG. 4. Distribution of locations of the iliac veins in relation to the Moro zones at the L4-5 disc space in patients with (left) and without (right) LSTV. Figure is available in color online only.

In patients without LSTV, the relationship of the left psoas muscle to the Moro zones was as follows: none (no apposition) in 17.9\%; Zone A and II in 7.1\%; Zones A and III in 3.6\%; Zones I and II in 7.1\%; Zones I, II, and III in $35.7 \%$; Zones II and III in $21.4 \%$; and Zone III only in $7.1 \%$ (Fig. 5C). The relationship with respect to the right psoas muscle in patients without LSTV was as follows: none (no apposition) in 7.1\%; none and Zone A (no apposition and anterior to the disc) in 7.1\%; Zones A and II in 7.1\%; Zones I and II in 10.7\%; Zones I, II, and III in 21.4\%; Zone II only in 3.6\%; Zones II and III in 35.7\%; and Zone III only in $7.1 \%$ (Fig. 5D).

In general, the psoas musculature in the LSTV patients was found to be separated from the vertebral column and disc, with the vasculature residing in this space (representative images shown in Fig. 6 upper). This is in contrast to the control group, where the vessels did not occupy the "safe corridor" typically used in a lateral transpsoas approach (Fig. 6 lower).

\section{Discussion}

Minimally invasive lumbar spine surgery continues to evolve and has many advantages over traditional open surgery, including less tissue trauma and blood loss, less postoperative pain, and shorter hospital stays with a quicker return to daily activities of living. ${ }^{20}$ Extreme lateral interbody fusion was first introduced by Pimenta in 2001 ("Lateral endoscopic transpsoas retroperitoneal approach for lumbar spine surgery," paper presented at the VIII Brazilian Spine Society Meeting, May 2001) and was shown in a subsequent study by Pimenta and colleagues (Ozgur et al. ${ }^{16}$ ) to be a safe and effective alternative approach to lum- bar fusion that avoids large anterior vascular structures, the nerves, the posterior paraspinal musculature, and the tension band.

The lateral approach to the L4-5 disc space, via the lateral transpsoas, poses a risk of injury to the nerves of the lumbar plexus and the vascular structures. Anatomical cadaveric dissection studies examined the safe anatomical zones where the neural and vascular structures would be avoided at the disc spaces from L-1 to L-5, and when we looked specifically at the L4-5 disc space, the midpoint of the disc at the junction of Moro Zone II and Zone III was deemed the safe zone. Interestingly, the dissection studies did not find any major vessels in Zones I, II, III, or IV at the L4-5 disc level. ${ }^{21}$ In comparison with the higher levels of L1-3, the more anteriorly located position of the lumbar plexus and the more posterior position of the vascular structures reduces the safe working zone at the L4-5 level to approximately $38 \%$ at L4-5 compared with $75 \%$ at L1-2.11

In our study population, the lumbosacral transitional group's iliac crest was found on average $12 \mathrm{~mm}$ above the middle of the L4-5 disc space whereas in the nontransitional group the iliac crest was found on average $4 \mathrm{~mm}$ below the middle of the L4-5 disc space and this finding was statistically significant. The implications in accessing the L4-5 disc in LSTV patients via a lateral transpsoas approach are evident since the iliac crest would present a real obstacle. Positioning techniques such as a bending the table may help, or the use of angled instruments may overcome this issue but would increase the complexity and risk profile of the surgery.

In the LSTV group, the level at which the iliac veins joined to become the IVC was on average $8 \mathrm{~mm}$ above the middle of the L4-5 disc space while for the nontransition- 

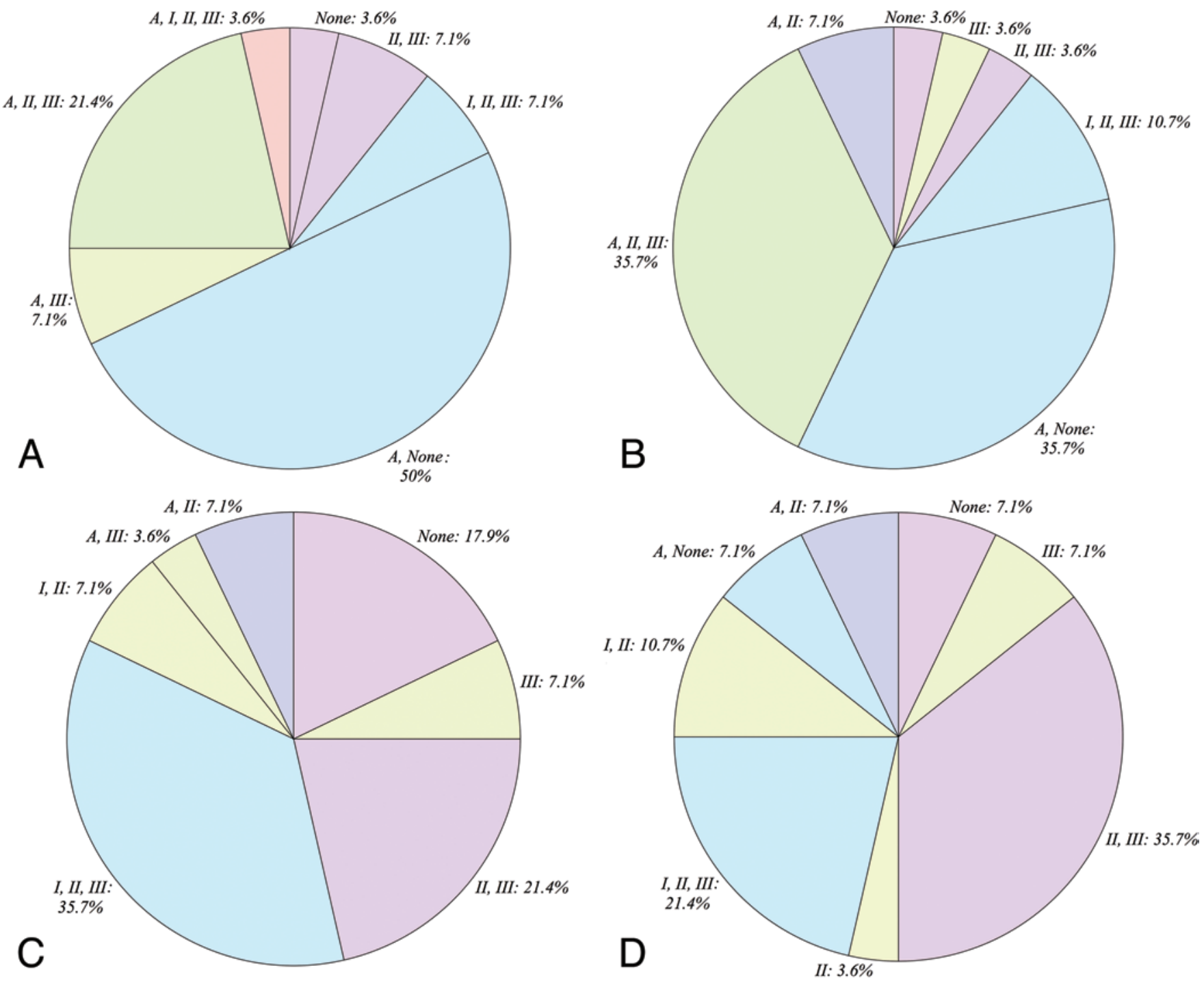

FIG. 5. Distribution of locations of the left (A and $\mathbf{C})$ and right (B and $\mathbf{D})$ psoas muscles in relation to the Moro zones at the $L 4-5$ disc space in patients with (A and B) and without (C and D) LSTV. Figure is available in color online only.

al group the iliac veins merged $2.6 \mathrm{~mm}$ below the middle of the L4-5 disc space on average, which was statistically significant. In the LSTV group, the iliac artery bifurcates on average $23 \mathrm{~mm}$ above the center of the L4-5 disc space versus iliac artery bifurcation occurring on average 11.3 $\mathrm{mm}$ below the middle of the L4-5 disc space in nontransitional patients (statistically significant). When we consider these data in relation to a lateral transpsoas approach to the L4-5 disc space this indicates that the iliac veins are still in a more lateral position and are at an increased risk of injury from retractor blades or instrumentation. ${ }^{3,5} \mathrm{In}$ addition, the safe working zone that was already known to be narrow at the L4-5 level is further reduced in the lumbosacral transitional patient. The psoas muscle in patients with LSTV is often not in the typical location next to the vertebral body and disc at the L4-5 level as shown in Fig. 5A and B compared with nontransitional patients depicted in Fig. 5C and D. This allows the iliac vessels to occupy the presumed safe zones, putting the patient at risk for a potential catastrophic vascular injury. ${ }^{23}$ In the LSTV group, the psoas muscle is anterior and not apposed to the L4-5 disc space $39.3 \%$ of the time on the right side and $53.6 \%$ of the time on the left side, with safe zone (Zone II or III) apposition found $14.3 \%$ of the time on the right and $14.2 \%$ of the time on the left at the L4-5 disc space. In contrast, the nontransitional patients had the expected psoas anatomy more frequently, with safe zone apposition found $67.8 \%$ of the time on both the right and left side of the L4-5 disc space. These findings support the potential higher risk of neurovascular injury of a lateral transpsoas approach in patients with LSTV.

Anterior approaches to the lumbar spine provide a large surface area for interbody fusion and indirect decompression of the neural foramina. Anterior exposure of the L4-5 disc level often requires mobilization of the left common iliac vessels. Venous injuries are among the most feared complications of these procedures, and in patients with LSTV, the ascending lumbar vein courses over the disc 

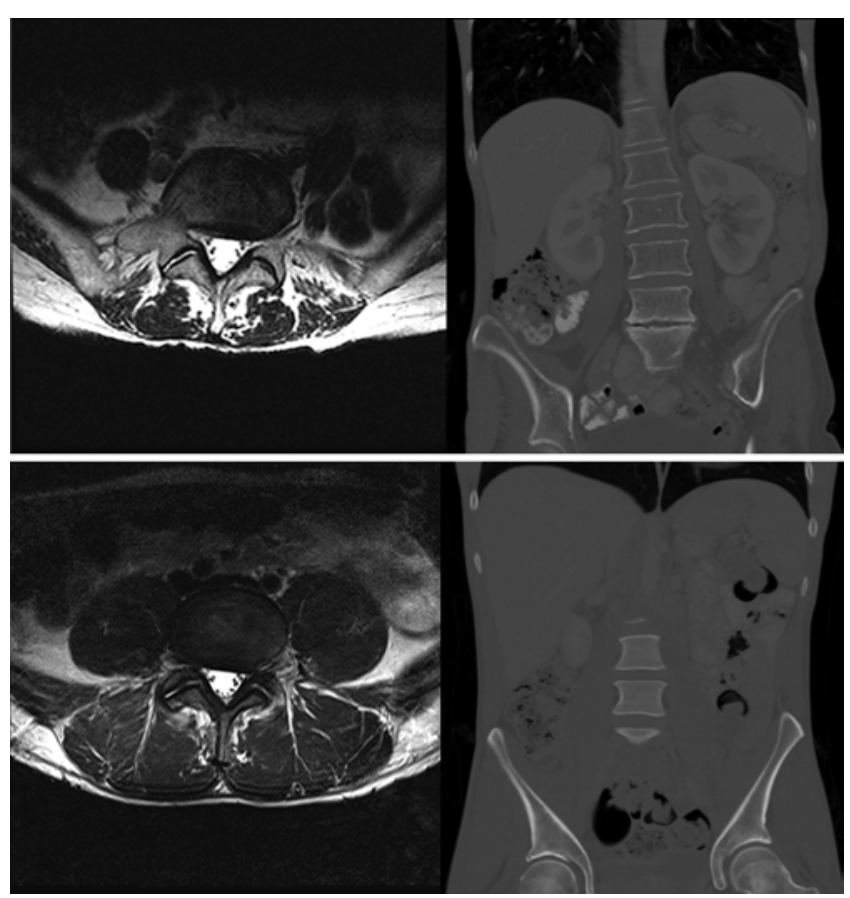

FIG. 6. Representative examples of axial T2-weighted MR images and coronal CT images obtained at the L4-5 disc level in a patient with a lumbosacral transitional vertebra (upper) and a patient from the control group (lower). Note that the psoas muscle is located more laterally and ventrally in the images obtained from the patient in the LSTV group rather than being apposed to the disc and vertebral body as they are in the patient without a lumbosacral transitional vertebra. In the images obtained in the patient from the LSTV group, the iliac vessels on the right are in Zones I and II, whereas they are located anterior to the vertebral body and in a more typical safe zone in the patient without a transitional vertebra. Note also the difference in the level of the iliac crest in relation to the L4-5 disc space.

space into venous union and with the higher merger of the iliac veins, working through the bifurcation of the inferior vena cava effectively reduces the annulotomy window and the size of the graft that can be used. ${ }^{8}$

The unique anatomy of lumbosacral transitional patients increases their risk of vascular complications as noted in our study population. In the LSTV group we found that the iliac veins were in Zone I and partly in Zone II $7.1 \%$ of the time and completely in Zone II $7.1 \%$ of the time. This meant that $14 \%$ of the time the iliac veins were in the already narrowed safe zone of the L4-5 disc space, putting them at an increased risk for injury. In the control group, the iliac veins were completely anterior or partly entering Zone I to the L4-5 disc space with a frequency of 93\% and only found in Zone I 7.1\% of the time. The iliac veins were never found in Zone II in the controls.

\section{Conclusions}

This study evaluated the anatomical differences of neurovascular, psoas muscle, and iliac crest anatomy in relation to the L4-5 disc space in patients with LSTV and the implications of these differences for minimally invasive lumbar spine surgery. We found that patients with LSTV may have a higher risk of iatrogenic neurovascular injury at this level and the high position of the iliac crest with respect to the L4-5 disc and the smaller safe zone increases the technical difficulty for a lateral transpsoas approach. The lateral transpsoas approach with interbody fusion is a less invasive surgical procedure that can be relatively safe, but careful preoperative planning and patient selection are necessary to minimize the risk of catastrophic complications.

\section{References}

1. Andersson GB: Epidemiological features of chronic low-back pain. Lancet 354:581-585, 1999

2. Apazidis A, Ricart PA, Diefenbach CM, Spivak JM: The prevalence of transitional vertebrae in the lumbar spine. Spine J 11:858-862, 2011

3. Assina R, Majmundar NJ, Herschman Y, Heary RF: First report of major vascular injury due to lateral transpsoas approach leading to fatality. J Neurosurg Spine 21:794-798, 2014

4. Baliga S, Treon K, Craig NJA: Low back pain: current surgical approaches. Asian Spine J 9:645-657, 2015

5. Buric J, Bombardieri D: Direct lesion and repair of a common iliac vein during XLIF approach. Eur Spine J 25 (Suppl 1):89-93, 2016

6. Castellvi AE, Goldstein LA, Chan DP: Lumbosacral transitional vertebrae and their relationship with lumbar extradural defects. Spine (Phila Pa 1976) 9:493-495, 1984

7. Dar G, Peled N: The association between sacralization and spondylolisthesis. Anat Sci Int 89:156-160, 2014

8. Fantini GA, Pappou IP, Girardi FP, Sandhu HS, Cammisa FP Jr: Major vascular injury during anterior lumbar spinal surgery: incidence, risk factors, and management. Spine (Phila Pa 1976) 32:2751-2758, 2007

9. Farshad-Amacker NA, Lurie B, Herzog RJ, Farshad M: Is the iliolumbar ligament a reliable identifier of the L5 vertebra in lumbosacral transitional anomalies? Eur Radiol 24:26232630, 2014

10. Frymoyer JW: Back pain and sciatica. N Engl J Med 318:291-300, 1988

11. Guérin P, Obeid I, Gille O, Bourghli A, Luc S, Pointillart V, et al: Safe working zones using the minimally invasive lateral retroperitoneal transpsoas approach: a morphometric study. Surg Radiol Anat 33:665-671, 2011

12. Konin GP, Walz DM: Lumbosacral transitional vertebrae: classification, imaging findings, and clinical relevance. AJNR Am J Neuroradiol 31:1778-1786, 2010

13. Luoma K, Vehmas T, Raininko R, Luukkonen R, Riihimäki $\mathrm{H}$ : Lumbosacral transitional vertebra: relation to disc degeneration and low back pain. Spine (Phila Pa 1976) 29:200205, 2004

14. Moro T, Kikuchi SI, Konno SI, Yaginuma H: An anatomic study of the lumbar plexus with respect to retroperitoneal endoscopic surgery. Spine (Phila Pa 1976) 28:423-428, 2003

15. Otani K, Konno S, Kikuchi S: Lumbosacral transitional vertebrae and nerve-root symptoms. J Bone Joint Surg Br 83:1137-1140, 2001

16. Ozgur BM, Aryan HE, Pimenta L, Taylor WR: Extreme lateral interbody fusion (XLIF): a novel surgical technique for anterior lumbar interbody fusion. Spine J 6:435-443, 2006

17. Sekharappa V, Amritanand R, Krishnan V, David KS: Lumbosacral transition vertebra: prevalence and its significance. Asian Spine J 8:51-58, 2014

18. Tang M, Yang XF, Yang SW, Han P, Ma YM, Yu H, et al: Lumbosacral transitional vertebra in a population-based study of 5860 individuals: prevalence and relationship to low back pain. Eur J Radiol 83:1679-1682, 2014

19. Tureli D, Ekinci G, Baltacioglu F: Is any landmark reliable 
in vertebral enumeration? A study of 3.0-Tesla lumbar MRI comparing skeletal, neural, and vascular markers. Clin Imaging 38:792-796, 2014

20. Uddin OM, Haque R, Sugrue PA, Ahmed YM, El Ahmadieh TY, Press JM, et al: Cost minimization in treatment of adult degenerative scoliosis. J Neurosurg Spine 23:798-806, 2015

21. Uribe JS, Arredondo N, Dakwar E, Vale FL: Defining the safe working zones using the minimally invasive lateral retroperitoneal transpsoas approach: an anatomical study. J Neurosurg Spine 13:260-266, 2010

22. Vergauwen S, Parizel PM, van Breusegem L, Van Goethem JW, Nackaerts Y, Van den Hauwe L, et al: Distribution and incidence of degenerative spine changes in patients with a lumbo-sacral transitional vertebra. Eur Spine J 6:168-172, 1997

23. Voyadzis JM, Felbaum D, Rhee J: The rising psoas sign: an analysis of preoperative imaging characteristics of aborted minimally invasive lateral interbody fusions at L4-5. J Neurosurg Spine 20:531-537, 2014

\section{Disclosures}

The authors report no conflict of interest concerning the materials or methods used in this study or the findings specified in this paper.

\section{Author Contributions}

Conception and design: Josiah, Bhatia. Acquisition of data: all authors. Analysis and interpretation of data: Josiah, Bhatia. Drafting the article: Josiah. Critically revising the article: Josiah. Reviewed submitted version of manuscript: all authors. Approved the final version of the manuscript on behalf of all authors: Josiah. Statistical analysis: Josiah. Study supervision: Bhatia.

\section{Correspondence}

Darnell T. Josiah, Department of Neurological Surgery, University of Wisconsin School of Medicine and Public Health, 600 Highland Ave., K4/854 CSC, Madison, WI 53792-8660. email: josiah@neurosurgery.wisc.edu. 